\title{
Passivity-based Tracking Control of an Omnidirectional Mobile Robot Using Only One Geometrical Parameter
}

\author{
Chao Ren ${ }^{\mathrm{a}}$, Yutong Ding ${ }^{\mathrm{a}}$, Shugen $\mathrm{Ma}^{\mathrm{a}} \mathrm{b}, *$, Liang $\mathrm{Hu}^{\mathrm{c}}$, Xinshan $\mathrm{Zhu}^{\mathrm{a}}$ \\ ${ }^{a}$ School of Electrical and Information Engineering, Tianjin University, Tianjin 300072, China \\ ${ }^{b}$ Department of Robotics, Ritsumeikan University, Shiga 525-8577, Japan \\ ${ }^{c}$ School of Computer Science and Informatics, De Montfort University, The Gateway, Leicester, LE1 9BH, UK
}

\begin{abstract}
This paper presents an output feedback tracking control scheme for a three-wheeled omnidirectional mobile robot, based on passivity property and a modified generalized proportional integral (GPI) observer. The proposed control approach is practical from an implementation point of view, since only robot position information and one robot geometrical parameter (i.e., contact radius) are required. Firstly, a dynamic model is given and passivity property is analyzed. Then the controller is designed based on passivity property and a modified GPI observer. The design objective is to preserve the passivity property in the closed-loop system, which is conceptually different from the traditional control design methodology. Particularly, the designed control system takes full advantage of the robot natural damping forces. Therefore, only considerably small or non differential feedback is needed. In addition, theoretical analysis is given to show the closed-loop stability behavior. Finally, experiments are conducted to validate the effectiveness of the proposed control system design in both tracking accuracy and robustness.
\end{abstract}

Keywords: Omnidirectional Mobile Robot, Trajectory Tracking Control, Disturbance Observer, Passivity Property, Output Feedback Control

\section{Introduction}

Recently, omnidirectional mobile robots (OMRs) are becoming increasingly popular in many applications, especially those in narrow spaces with high maneuverability requirements, such as warehouses, factories and hospitals. This is due to the fact that OMRs can perform arbitrary motion in an arbitrary orientation without changing the direction of wheels [1].

OMRs are typical nonlinear, time-varying, multi-input-multioutput systems, subject to various external disturbances (i.g., uncertain payload, friction forces). In the literature, the dynamic modeling and control of OMRs have been studied by many researchers in recent two decades [2-11]. In [2], a feedback linearization approach, resolved acceleration control (RAC), was applied to an OMR with three lateral orthogonal-wheel assemblies, in which the control performance depends on the accuracy of the dynamic model. [3] designed a nonlinear controller for an OMR using a trajectory linearization control method with consideration of actuator dynamics. Considering static friction effects, [4] presented a dynamic model for an OMR and an experimental identification method to estimate the parameters of the static friction model. In [5], a model-predictive

\footnotetext{
^ This work was supported by the National Natural Science Foundation of China under Grant 61603270 and Tianjin Natural Science Foundation under Grant 18JCQNJC04600.

${ }^{*}$ Corresponding author

Email addresses: renchao@tju.edu.cn (Chao Ren), yutong_ding@tju.edu.cn (Yutong Ding), shugen.ma@ieee.org (Shugen Ma), liang.hu@dmu.ac.uk (Liang Hu), zhongyang. fei@hit.edu.cn (Xinshan Zhu)
}

control scheme with friction compensation using a static friction model was proposed for an OMR. Six parameters of the static friction model were identified using the approach proposed in [4]. However, the identification process is complex. In [6], a linear optimal controller was designed based on a simple linear model, which is obtained by linearizing a complicated dynamic model of a three-wheeled OMR using kinematics. In [7], a smooth switching adaptive sliding-mode controller was proposed to tackle the tracking tasks in the presence of both structured and unstructured uncertainties. The parameters of motor dynamics were assumed to be unknown. In [8], a switching quasi-linear-parameter-varying controller using LMI-based techniques was proposed for a four-wheeled OMR. Considering wheel switching, a continuous dynamic modeling and control approach of an OMR were discussed in [9] and [10], respectively. An observer was used to estimate the complicated dynamics, unmodeled dynamics and input-output cross-couplings in [10]. It should be mentioned that the designed control system in [10] depends on part of dynamic model information. In [11], quantitative feedback theory was applied to design a proportional-integral controller for robust low-level control for a redundant OMR with four wheels.

All of the controllers mentioned above are model-based design approaches, in which a complete dynamic model including motor dynamics (i.g., $[2-6,8,11]$ ) or at least part of the dynamic model information (i.g., $[7,10]$ ) is required. It is well known that both the dynamic modeling and parameter identification are complex and time-consuming in practice. Therefore, model-based control design may be restrictive from an imple- 
mentation point of view if a dynamic model or its parameters are unknown. From a practical point of view, a control system design will be particularly attractive if the tedious dynamic modeling and parameter identification process can be avoided.

Passivity is one of the most fundamental properties of robotic systems [12]. Passivity-based control has been a very powerful design concept in many control problems of robotics, i.g., robot control [13, 14], teleoperation control $[15,16]$, to name a few. On the other hand, generalized proportional integral (GPI) observer is a disturbance observer characterized by a high dimensional extension [17], and it thus has a good estimation performance of fast-varying disturbances. GPI observer based control has been successfully applied in various practical applications [18-20], to name a few. However, although GPI observer based control design does not require complete model information, part of the model information is still needed.

In this paper, a passivity-based output feedback control (POFC) is designed for trajectory tracking of a three-wheeled OMR. Firstly, a robot dynamic model is given and the passivity property of the robot is analyzed. Then the control system is designed, in which a modified GPI observer is employed to estimate disturbances and control errors. The design objective is to preserve the passivity property of the robot in the closed-loop system, which is conceptually different from traditional control design, such as feedback linearization control approaches. It should be emphasized that the differential feedback gains can be selected considerably small or even zero due to an effective exploitation of the robot natural damping. The resulting control system only requires one geometrical parameter (i.e., the contact radius of each wheel), without using any other model parameters. Theoretical analysis is presented to show the closedloop stability behavior. Finally, experiments are conducted to compare the tracking accuracy and robustness of the proposed control design against traditional model-based RAC.

The remainder of this paper is organized as follows. In Section II, dynamic modeling and analysis of a three-wheeled OMR are presented. The proposed passivity-based output feedback trajectory tracking control scheme as well as stability analysis is presented in Section III. In Section IV, implementation details, experimental results and discussions are presented. Finally, conclusions are drawn in Section V.

\section{Dynamic modeling and analysis}

In this section, a dynamic model for the robot prototype is given and then passivity property of the robot dynamic system is analyzed.

The prototype platform is shown in Fig. 1, with three MY wheel-II assemblies arranged at $120^{\circ}$ intervals beneath the steel disk. Each assembly is actuated with a DC motor. For a detailed description of the MY wheel-II mechanism and the prototype platform, the readers are referred to [21].

The two coordinate frames used in the modeling are shown in Fig. 2: the world coordinate frame $\{W\}$ fixed on the ground and the moving coordinate frame $\{M\}$ fixed on the robot geometric center. The nomenclature is defined in Table 1.

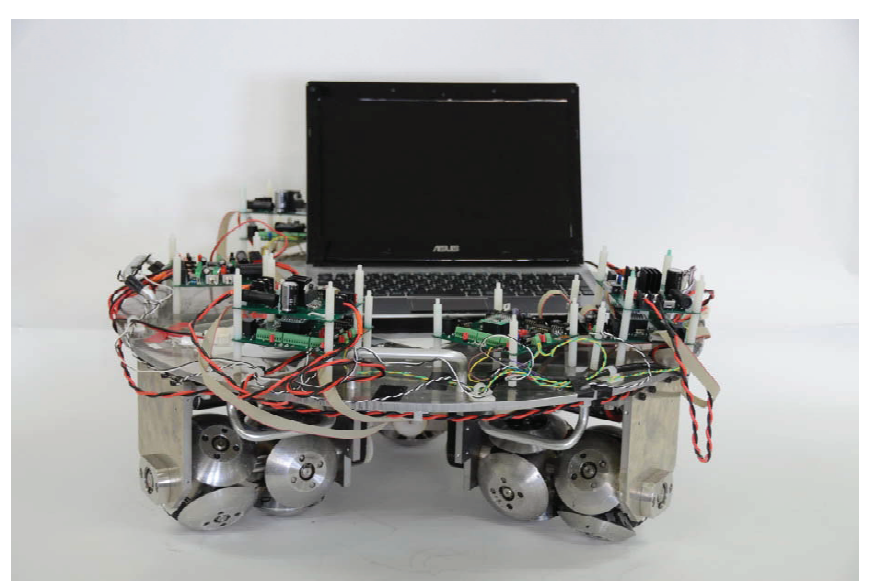

Figure 1: Prototype platform.

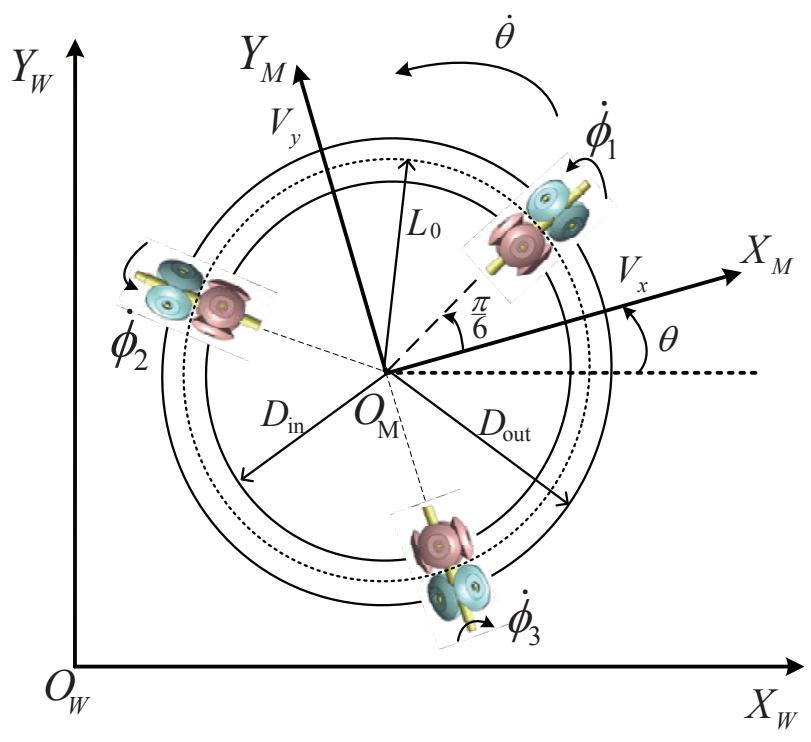

Figure 2: Coordinate frames of the omnidirectional mobile robot.

Note that, as shown in Fig. 1 and Fig. 2, each MY wheel-II assembly has two contact points with the ground, and thereby two contact radiuses exist for each wheel (i.e., $D_{\text {in }}$ and $D_{\text {out }}$ ) [22]. In this paper, the average contact radius (i.e., $L_{0}=\left(D_{i n}+\right.$ $\left.D_{\text {out }}\right) / 2$ ) is used in the dynamic model. In addition, it is assumed that no slippage is between the wheel and the motion surface. Part of the friction forces in the transmission system (e.g. coulomb friction), dead-zone and backlash, and friction forces between each passive wheel and ground are unmodeled.

The robot dynamic model in the world coordinate frame including motor dynamics is given as follows [23]:

$$
M \ddot{q}+C \dot{q}+D \dot{q}=\tau,
$$

where $\boldsymbol{\tau}=\boldsymbol{B} \boldsymbol{u}$, which is considered as the virtual control input in this paper, and the real control input, $\boldsymbol{u}=\left[\begin{array}{lll}u_{1} & u_{2} & u_{3}\end{array}\right]^{T}$, is the 
Table 1: Nomenclature

\begin{tabular}{|c|c|c|}
\hline \multicolumn{3}{|c|}{ World coordinate frame } \\
\hline$q=$ & $\left.\begin{array}{lll}x & y & \theta\end{array}\right]^{T}$ & Robot position and orientation angle \\
\hline $\boldsymbol{V}_{M}=$ & $\left.\begin{array}{lll}V_{x} & V_{y} & \dot{\theta}\end{array}\right]^{T}$ & $\begin{array}{l}\text { Robot translational velocity and } \\
\text { rotational angular rate }\end{array}$ \\
\hline \multicolumn{3}{|c|}{ Mechanical constants } \\
\hline & $m$ & Robot mass \\
\hline & $I_{v}$ & $\begin{array}{l}\text { Robot moment of inertia around } \\
\text { the mass center of the robot }\end{array}$ \\
\hline & $I_{w}$ & $\begin{array}{c}\text { Wheel moment of inertia around } \\
\text { the wheel shaft }\end{array}$ \\
\hline & $r$ & Wheel radius \\
\hline & $D_{\text {in }}$ & Inner contact radius \\
\hline & $D_{\text {out }}$ & Outer contact radius \\
\hline & $L_{0}$ & Average contact radius \\
\hline & $I_{0}$ & $\begin{array}{l}\text { Combined moment of inertia of } \\
\text { motor, gear train and wheel referred } \\
\text { to the motor shaft }\end{array}$ \\
\hline & $b_{0}$ & $\begin{array}{l}\text { Combined viscous friction coefficient } \\
\text { of the motor, gear and wheel shaft. }\end{array}$ \\
\hline & $k_{b}$ & Motor back EMF constant \\
\hline & $k_{t}$ & Motor torque constant \\
\hline & $R_{a}$ & Motor armature resistance \\
\hline & $n$ & Gear reduction ratio \\
\hline
\end{tabular}

supplied voltage of three motors; and

$$
\begin{aligned}
& \boldsymbol{M}=\frac{1}{p_{2}}\left[\begin{array}{ccc}
\frac{3}{2} p_{0}+m & 0 & 0 \\
0 & \frac{3}{2} p_{0}+m & 0 \\
0 & 0 & 3 p_{0} L_{0}^{2}+I_{v}
\end{array}\right] \\
& \boldsymbol{C}=\frac{1}{p_{2}}\left[\begin{array}{ccc}
0 & \frac{3}{2} p_{0} \dot{\theta} & 0 \\
-\frac{3}{2} p_{0} \dot{\theta} & 0 & 0 \\
0 & 0 & 0
\end{array}\right] \\
& \boldsymbol{D}=\frac{1}{p_{2}}\left[\begin{array}{ccc}
\frac{3}{2} p_{1} & 0 & 0 \\
0 & \frac{3}{2} p_{1} & 0 \\
0 & 0 & 3 p_{1} L_{0}^{2}
\end{array}\right] \\
& \boldsymbol{B}=\frac{1}{2}\left[\begin{array}{ccc}
-\cos \theta-\sqrt{3} \sin \theta & -\cos \theta+\sqrt{3} \sin \theta & 2 \cos \theta \\
-\sin \theta+\sqrt{3} \cos \theta & -\sin \theta-\sqrt{3} \cos \theta & 2 \sin \theta \\
2 L_{0} & 2 L_{0} & 2 L_{0}
\end{array}\right],
\end{aligned}
$$

$p_{0}=\frac{n^{2} I_{0}}{r^{2}}, p_{1}=\frac{n^{2}}{r^{2}}\left(b_{0}+\frac{k_{t} k_{b}}{R_{a}}\right), p_{2}=\frac{n k_{t}}{r R_{a}} . \quad \boldsymbol{D} \dot{\boldsymbol{q}}$ is the dissipative force, due to the combined viscous friction of the motor, gear and wheel shaft, as well as the motor armature resistance, gear reduction, etc. It relates to the loss or dissipation of energy. Since $\boldsymbol{D}$ is positive definite, the robot is a fully damped system. On the other hand, according to the standard passivity definition, (1) defines an output strictly passive mapping from the virtual control input $\boldsymbol{\tau}$ to $\dot{\boldsymbol{q}}$. Note that, the passive mapping from the real control input $\boldsymbol{u}$ to $\dot{\boldsymbol{q}}$ cannot be guaranteed.
Property 1. $M$ is a symmetric positive definite matrix, it satisfies $\lambda_{\min }(\boldsymbol{M})\|\boldsymbol{x}\|^{2} \leq \boldsymbol{x}^{T} \boldsymbol{M} \boldsymbol{x} \leq \lambda_{\max }(\boldsymbol{M})\|\boldsymbol{x}\|^{2}, \forall \boldsymbol{x} \in \mathbb{R}^{3}$, where $\|\cdot\|$ denotes the Euclidean norm; $\lambda_{\min }(\cdot)$ and $\lambda_{\max }(\cdot)$ denote the minimum and maximum eigenvalue, respectively.

Property 2. With the definition of $\boldsymbol{M}$ and $\boldsymbol{C}$ in (1), $\dot{\boldsymbol{M}}-2 \boldsymbol{C}$ is skew symmetric.

Remark 1. The matrix $\boldsymbol{D}$ can be rewritten as follows:

$$
\boldsymbol{D}=\frac{n R_{a}}{r k_{t}}\left(b_{0}+\frac{k_{t} k_{b}}{R_{a}}\right)\left[\begin{array}{ccc}
\frac{3}{2} & 0 & 0 \\
0 & \frac{3}{2} & 0 \\
0 & 0 & 3 L_{0}^{2}
\end{array}\right] .
$$

It can be seen that, the robot natural damping is related to the gear reduction ratio and wheel radius. More specifically, the robot natural damping has a positive correlation with the gear reduction ratio $n$ and an inverse correlation with the wheel radius $r$.

\section{Passivity-based control}

\subsection{Control system design}

Assuming that the robot dynamic equation (1) is known, the well-known passivity-based trajectory tracking controller can be applied as follows [24]:

$$
\boldsymbol{\tau}=\boldsymbol{M} \ddot{\boldsymbol{q}}_{r}+(\boldsymbol{C}+\boldsymbol{D}) \dot{\boldsymbol{q}}_{r}-\boldsymbol{K}_{d} \boldsymbol{s},
$$

where $\dot{\boldsymbol{q}}_{r}=\dot{\boldsymbol{q}}_{d}-\boldsymbol{\Lambda} \boldsymbol{e}, \boldsymbol{q}_{d}=\left[\begin{array}{lll}x_{d} & y_{d} & \theta_{d}\end{array}\right]^{T}$ is the desired robot trajectory, $\boldsymbol{e}=\boldsymbol{q}-\boldsymbol{q}_{d}, \boldsymbol{s}=\dot{\boldsymbol{e}}+\boldsymbol{\Lambda} \boldsymbol{e} ; \boldsymbol{K}_{d}$ and $\boldsymbol{\Lambda} \in \mathbb{R}^{3 \times 3}$ are diagonal positive definite matrices. The term $\boldsymbol{K}_{d} \boldsymbol{s}$ represents the damping injection.

Combining (1) and (2), the closed-loop error dynamics can be obtained as follows:

$$
M \dot{s}+C s+D s+K_{d} s=0 .
$$

Based on Lyapunov stability analysis, it is straightforward to obtain that $s \rightarrow 0$ as $t \rightarrow \infty$, and thus $\boldsymbol{e} \rightarrow 0, \dot{\boldsymbol{e}} \rightarrow 0$ as $t \rightarrow \infty$ [24].

It is clear that the energy minimum of open-loop system (1), i.e., $(\boldsymbol{q}, \dot{\boldsymbol{q}})=(0,0)$, has been shifted to $(\boldsymbol{e}, \dot{\boldsymbol{e}})=(0,0)$ by the controller (2). Note that the total energy of the open-loop system (1) is

$$
\boldsymbol{H}_{0}(\boldsymbol{q}, \dot{\boldsymbol{q}})=\frac{1}{2} \dot{\boldsymbol{q}}^{T} \boldsymbol{M} \dot{\boldsymbol{q}}
$$

The controller (2) actually reshapes the original open-loop energy function of (4) into

$$
\boldsymbol{H}_{1}(\boldsymbol{e}, \dot{\boldsymbol{e}})=\frac{1}{2} \boldsymbol{s}^{T} \boldsymbol{M} \boldsymbol{s}
$$

which is the total energy of the closed-loop system (3). This is the well-known energy shaping plus damping ideas of the passivity-based control approach [25].

Consider the case that the matrix $\boldsymbol{M}, \boldsymbol{C}$ and $\boldsymbol{D}$ in (1) are completely unknown and only the input matrix $\boldsymbol{B}$ is known. To 
maintain the passivity property, the objective is to design a controller which results in a closed-loop system maintaining the similar structure of (3).

Then the open-loop tracking error dynamics can be written as:

$$
\ddot{\boldsymbol{e}}=\ddot{\boldsymbol{q}}-\ddot{\boldsymbol{q}}_{d}=-\boldsymbol{\Lambda} \dot{\boldsymbol{e}}+\boldsymbol{M}^{-1}(\tau-C s-D s)+f,
$$

where $\boldsymbol{f}=-\boldsymbol{M}^{-1}\left(\boldsymbol{C} \dot{\boldsymbol{q}}_{r}+\boldsymbol{D} \dot{\boldsymbol{q}}_{r}\right)-\ddot{\boldsymbol{q}}_{r}+\boldsymbol{\xi}(t)$, is the time-varying perturbation input vector, and $\boldsymbol{\xi}(t)$ represents the modeling errors, including ummodeled forces, parameter uncertainties and external disturbances. Defining $\boldsymbol{w}=\boldsymbol{M} \boldsymbol{f}$, then the following assumption is made.

Assumption: The time-varying perturbation input vector $\boldsymbol{w}$ can be approximated by a $(p-1)$-degree family of Taylor timepolynomial inputs and a residual term; that is,

$$
\boldsymbol{w}(t) \approx \sum_{i=0}^{p-1} \boldsymbol{\alpha}_{i} t^{i}+\boldsymbol{\delta}(t),
$$

with $\alpha_{i} \in \mathbb{R}^{3 \times 3}$ being constant coefficients, and the $k t h$ time derivatives of the residual term $\delta(t)$, i.e., $\boldsymbol{\delta}^{(k)}(t)(k \geqslant p)$, being uniformly absolutely bounded. Thus $\boldsymbol{w}^{(p)}(t)=\boldsymbol{\delta}^{(p)}(t)$.

Define $\boldsymbol{e}_{1}=\boldsymbol{e}, \boldsymbol{e}_{2}=\dot{\boldsymbol{e}}$ and $\boldsymbol{w}_{1}=\boldsymbol{w}$. Then the open-loop tracking error dynamics (6) can be rewritten in state space as

$$
\begin{aligned}
& \dot{\boldsymbol{e}}_{1}=\boldsymbol{e}_{2}, \\
& \dot{\boldsymbol{e}}_{2}=-\boldsymbol{\Lambda} \dot{\boldsymbol{e}}+\boldsymbol{M}^{-1}(\boldsymbol{\tau}-\boldsymbol{C} \boldsymbol{s}-\boldsymbol{D} \boldsymbol{s})+\boldsymbol{M}^{-1} \boldsymbol{w}_{1}, \\
& \dot{\boldsymbol{w}}_{1}=\boldsymbol{w}_{2}, \\
& \vdots \\
& \dot{\boldsymbol{w}}_{p-1}=\boldsymbol{w}_{p}, \\
& \dot{\boldsymbol{w}}_{p}=\boldsymbol{\delta}^{(p)}(t),
\end{aligned}
$$

Assuming that $\boldsymbol{M}=\boldsymbol{I}$, then $\boldsymbol{C}(\dot{\boldsymbol{q}})=\mathbf{0}$ is satisfied if it is defined using the Christoffel sysmbols $[12,26]$. Define $\hat{\boldsymbol{e}}_{1}$ and $\hat{\boldsymbol{e}}_{2}$ as the estimation of $\boldsymbol{e}_{1}$ and $\boldsymbol{e}_{2}$, respectively. Then a modified GPI observer can be derived for the error dynamics (8) as follows [26]:

$$
\begin{aligned}
& \dot{\hat{\boldsymbol{e}}}_{1}=\hat{\boldsymbol{e}}_{2}-\boldsymbol{\Lambda} \hat{\boldsymbol{e}}_{1}+\left(\lambda_{p+1}-\boldsymbol{\Lambda}\right) \tilde{\boldsymbol{e}}, \\
& \dot{\hat{\boldsymbol{e}}}_{2}=\lambda_{p} \tilde{\boldsymbol{e}}, \\
& \dot{\hat{\boldsymbol{w}}}_{1}=\hat{\boldsymbol{w}}_{2}+\lambda_{p-1} \tilde{\boldsymbol{e}}, \\
& \vdots \\
& \dot{\hat{\boldsymbol{w}}}_{p-1}=\hat{\boldsymbol{w}}_{p}+\lambda_{1} \tilde{\boldsymbol{e}}, \\
& \dot{\hat{\boldsymbol{w}}}_{p}=\lambda_{0} \tilde{\boldsymbol{e}},
\end{aligned}
$$

where $\tilde{\boldsymbol{e}}=\boldsymbol{e}-\hat{\boldsymbol{e}}_{1}$, the observer gains $\lambda_{i} \in \mathbb{R}^{3 \times 3}, i=0,1, \ldots p+1$ are diagonal positive definite. $\lambda_{i j}$ is used to represent the jth diagonal element in $\lambda_{i}, j=1,2,3 . \hat{\boldsymbol{w}}_{i}$ is the estimation of $\boldsymbol{w}_{i}, i=$ $1,2, \ldots p \cdot p$ is the order of GPI observer.

The selection of $\lambda_{i}$ is to make sure that the poles of the observer error dynamics in three channels are placed at the desired locations. The characteristic polynomial of the observer error dynamics for each channel is as follows:

$$
\eta\left(s_{j}\right)=s_{j}^{p+2}+\lambda_{(p+1) j} s_{j}^{p+1}+\cdots+\lambda_{1 j} s_{j}+\lambda_{0 j},
$$

where $j=1,2,3$. With proper selection of $\boldsymbol{\lambda}_{i}, \dot{\boldsymbol{e}} \approx \dot{\hat{\boldsymbol{e}}}_{1}$ and $\boldsymbol{w} \approx \hat{\boldsymbol{w}}_{1}$.

For the sake of simplicity, the poles in the three channels are placed in the following manner:

$$
\eta\left(s_{j}\right)=\left(s_{j}+\omega_{o j}\right)^{p+2},
$$

where $j=1,2,3, \omega_{o j}\left(\omega_{o j}>0\right)$ is a parameter to specify the desired poles. In this paper, the observer order $p$ is set as 2 , and then the observer gains can be easily obtained by comparing (10) and (11) as follows:

$$
\begin{gathered}
\lambda_{0}=\left[\begin{array}{lll}
\omega_{o 1}^{4} & & \\
& \omega_{o 2}^{4} & \\
& & \omega_{o 3}^{4}
\end{array}\right], \lambda_{1}=\left[\begin{array}{ccc}
4 \omega_{o 1}^{3} & & \\
& 4 \omega_{o 2}^{3} & \\
& & 4 \omega_{o 3}^{3}
\end{array}\right], \\
\lambda_{2}=\left[\begin{array}{lll}
6 \omega_{o 1}^{2} & & \\
& 6 \omega_{o 2}^{2} & \\
& & 6 \omega_{o 3}^{2}
\end{array}\right], \lambda_{3}=\left[\begin{array}{lll}
4 \omega_{o 1} & \\
& 4 \omega_{o 2} & \\
& & 4 \omega_{o 3}
\end{array}\right] .
\end{gathered}
$$

The design objective is to derive a controller resulting in a similar structure of (3), in order to preserve the passivity property in the closed-loop system. Therefore, a possible selection of the controller can be given as:

$$
\boldsymbol{\tau}=-\boldsymbol{K}_{d}\left(\dot{\hat{\boldsymbol{e}}}_{1}+\boldsymbol{\Lambda} \boldsymbol{e}\right)-\hat{\boldsymbol{w}}_{1}=-\boldsymbol{K}_{d} \boldsymbol{s}+\boldsymbol{K}_{d} \dot{\tilde{\boldsymbol{e}}}-\hat{\boldsymbol{w}}_{1},
$$

where $\dot{\tilde{\boldsymbol{e}}}=\dot{\boldsymbol{e}}-\dot{\hat{\boldsymbol{e}}}_{1}$.

With the selection of (12), the closed-loop tracking error dynamic equation can be obtained by combining (6) and (12):

$$
\boldsymbol{M} \dot{s}+C s+D s+K_{d} s=\sigma .
$$

where $\boldsymbol{\sigma}=\boldsymbol{K}_{d} \dot{\tilde{\boldsymbol{e}}}+\tilde{\boldsymbol{w}}$ and $\tilde{\boldsymbol{w}}=\boldsymbol{w}-\hat{\boldsymbol{w}}_{1}$. It is noted that the closedloop tracking error equation (13) has a similar structure with (3). It can be proved that system (13) is strictly passive from $\sigma$ to $s$.

Finally, since $\tau$ is a virtual control input, the real control input of the proposed trajectory tracking controller is given as follows:

$$
\mathbf{u}=\boldsymbol{B}^{-1}\left(-\boldsymbol{K}_{d}\left(\dot{\hat{\boldsymbol{e}}}_{1}+\boldsymbol{\Lambda} \boldsymbol{e}\right)-\hat{\boldsymbol{w}}_{1}\right)
$$

It can be seen from (9) and (14) that the only required model parameter in implementation of the designed control system is the contact radius $L_{0}$, which is the geometrical information of robot. Compared with traditional model-based control design, such as RAC in [2], the tedious dynamic modeling and parameter identification process can be avoided. As a result, the proposed control design may be an attractive option from an implementation point of view. In addition, it can be seen in the observer (9) and controller (14) that only the position feedback signal is needed without measuring the robot velocity.

Remark 2. As already mentioned before, since the robot prototype is a fully damped dynamic system, the damping injection may be avoided by exploiting the robot natural damping. In other words, $\boldsymbol{K}_{d}$ may be set quite small or even zero in practice because of an effective exploitation of the robot natural damping. This design concept is totally different from traditional control design approach, such as RAC in [2]. In [2], the robot natural damping is canceled in the feedback linearization and $\boldsymbol{K}_{d}$ has to be set large enough to ensure system stability. It should be pointed out that high frequency disturbance may be introduced into the control system due to large $\boldsymbol{K}_{d}$. 


\subsection{Stability analysis}

The boundedness of $\dot{\tilde{\boldsymbol{e}}}$ and $\tilde{\boldsymbol{w}}$ have been proved in [26], and thus $\sigma$ is bounded.

Theorem 1. The closed-loop system (13) is bounded-input bounded-output (BIBO) stable with respect to the pair $(s, \sigma)$, i.e., $\exists \beta_{1}, \beta_{2}>0$ such that

$$
\|\boldsymbol{s}\| \leq \beta_{1}+\beta_{2}\|\sigma\|
$$

and $\boldsymbol{e} \in \mathcal{L}_{\infty}^{3}, \dot{\boldsymbol{e}} \in \mathcal{L}_{\infty}^{3}$.

Proof. Consider a Lyapunov function:

$$
\mathrm{V}(t)=\frac{1}{2} \boldsymbol{s}^{T} \boldsymbol{M} \boldsymbol{s}
$$

According to Property 1, the Lyapunov function (16) satisfies:

$$
\frac{1}{2} \lambda_{\min }(\boldsymbol{M})\|\boldsymbol{s}\|^{2} \leq V \leq \frac{1}{2} \lambda_{\max }(\boldsymbol{M})\|\boldsymbol{s}\|^{2} .
$$

The derivative of (16) can be derived as follows:

$$
\begin{aligned}
& \dot{V}=s^{T} M \dot{s}+\frac{1}{2} s^{T} \dot{M} s \\
& =s^{T}\left(\sigma-C s-D s-K_{d} s\right)+\frac{1}{2} s^{T} \dot{M} s \\
& =\boldsymbol{s}^{T} \sigma+\frac{1}{2} \boldsymbol{s}^{T}(\dot{M}-2 C) s-s^{T}\left(D+K_{d}\right) s \\
& =\boldsymbol{s}^{T} \sigma-\boldsymbol{s}^{T}\left(D+K_{d}\right) s \text {. }
\end{aligned}
$$

Note that Property 2 has been used in (18).

Combining (17) and (18), the following inequation is gained:

$$
\begin{aligned}
\dot{V} \leq & -\frac{2 \lambda_{\min }\left(\boldsymbol{D}+\boldsymbol{K}_{d}\right)}{\lambda_{\max }(\boldsymbol{M})} V+\sqrt{\frac{2 \mathrm{~V}}{\lambda_{\min }(\boldsymbol{M})}}\|\boldsymbol{\sigma}\| \\
& =-\gamma_{1} \mathrm{~V}+\sqrt{\frac{2 \mathrm{~V}}{\lambda_{\min }(\boldsymbol{M})}}\|\boldsymbol{\sigma}\|,
\end{aligned}
$$

where $\gamma_{1}=\frac{2 \lambda_{\min }\left(\boldsymbol{D}+\boldsymbol{K}_{d}\right)}{\lambda_{\max }(\boldsymbol{M})}>0$. Divide both sides of (19) by $2 \sqrt{V}$ :

$$
\frac{\dot{V}}{2 \sqrt{V}}=\frac{d}{d t}(\sqrt{V}) \leq-\frac{\gamma_{1}}{2} \sqrt{V}+\sqrt{\frac{1}{2 \lambda_{\min }(\boldsymbol{M})}}\|\sigma\| .
$$

Define $H=\sqrt{V}, \gamma=\frac{\gamma_{1}}{2}>0, \varepsilon=\sqrt{\frac{1}{2 \lambda_{\min }(M)}}\|\sigma\|$, then $(20)$ can be rewritten as:

$$
\dot{H} \leq-\gamma H+\varepsilon .
$$

Then there is:

$$
H(t) \leq H(0) \exp (-\gamma t)+\frac{\varepsilon}{\gamma}[1-\exp (-\gamma t)], \forall t \in[0, \infty) .
$$

Since $-\gamma t \leq 0,0<\exp (-\gamma t) \leq 1$, inequation (22) can be written as follows by combining with (17):

$$
\sqrt{\frac{\lambda_{\min }(\boldsymbol{M})}{2}}\|s\| \leq H \leq H(0)+\frac{\varepsilon}{\gamma} .
$$

Then the following can be obtained:

$$
\|\boldsymbol{s}\| \leq \beta_{1}+\beta_{2}\|\sigma\|,
$$

where $\beta_{1}=\sqrt{\frac{2}{\lambda_{\min }(\boldsymbol{M})}} H(0), \beta_{2}=\frac{1}{\gamma \lambda_{\min }(\boldsymbol{M})}$.

Since $\boldsymbol{s}=\dot{\boldsymbol{e}}+\boldsymbol{\Lambda} \boldsymbol{e}$, and $\boldsymbol{\Lambda} \in \mathbb{R}^{3 \times 3}$ is diagonal positive definite, it can be easily conclude that if $s \in \mathcal{L}_{\infty}^{3}$, then $\boldsymbol{e} \in \mathcal{L}_{\infty}^{3}, \dot{\boldsymbol{e}} \in \mathcal{L}_{\infty}^{3}$.

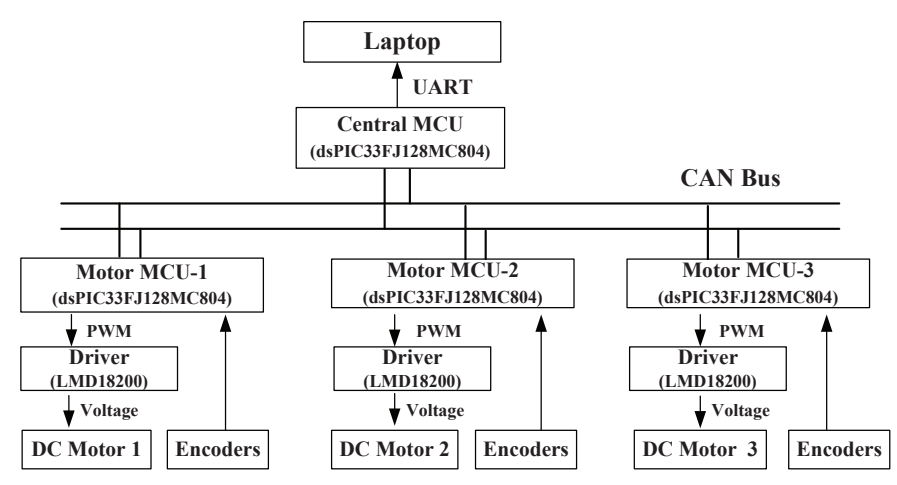

Figure 3: Schematic of the experimental setup.

\section{Experiments}

Fig. 3 shows the complete schematic of the experimental setup. The central controller and motor controllers are simple microcontrollers (all are dsPIC33FJ128MC804 from Microchip). They were programmed to operate at 40 million instructions per second. Three DC motors (Maxon) are the same with gear reduction ratio of 185.7 and the nominal voltage of 24 $\mathrm{V}$. Each motor is installed with an incremental encoder (1024 counts per turn, Maxon). Three DC motor drivers (LMD18200 from Texas Instruments) are identical.

As seen from Fig. 3, the communication between the central controller and the three motor controllers is via CAN bus, which was programmed to operate at $1 \mathrm{Mb} / \mathrm{s}$. The central controller calculates the control law and generates the control input to three motor controllers. Then each motor controller generates the corresponding pulse-width modulation (PWM) signal to each motor driver. In addition, each motor controller collects information from encoder and then sends it to the central controller via CAN bus. The robot posture is determined using odometry which is commonly used in the tracking control study of mobile robot $[27,28]$, to name a few. The laptop is used to receive experimental data via UART. The sample time is set as $10 \mathrm{~ms}$.

\subsection{Tracking performance}

In this part, experiments were conducted to compare the tracking performances of the proposed design against traditional model-based RAC in [2]. The parameters of the robot prototype were identified as follows: $m=35 \mathrm{~kg}, I_{v}=1.35 \mathrm{~kg} \cdot \mathrm{m}^{2}$, $r=0.06 \mathrm{~m}, D_{\text {in }}=0.147 \mathrm{~m}, D_{\text {out }}=0.236 \mathrm{~m}, L_{0}=0.192 \mathrm{~m}, I_{0}=$ $3.15 \times 10^{-5} \mathrm{~kg} \cdot \mathrm{m}^{2}, k_{t}=0.0292 \mathrm{~N} \cdot \mathrm{m} / \mathrm{A}, k_{b}=328 \mathrm{rpm} / \mathrm{V}, n=$ $186, b_{0}=1.5 \times 10^{-4} \mathrm{Nms} / \mathrm{rad}, R_{a}=0.61 \Omega$. It should be reminded that only the geometrical parameter $L_{0}$ is needed in the proposed control design without requiring any other model parameters.

The controller parameters of RAC were set as:

$$
\boldsymbol{K}_{p}=\left[\begin{array}{lll}
16 & & \\
& 16 & \\
& & 16
\end{array}\right], \quad \boldsymbol{K}_{d}=\left[\begin{array}{lll}
40 & & \\
& 40 & \\
& & 40
\end{array}\right] .
$$


Table 2: Experimental results: IAE of RAC and POFC

\begin{tabular}{ccccc} 
Index & $I A E_{x y}(\mathrm{~m})$ & $I A E_{\theta}(\mathrm{rad})$ & $M A E_{x y}(\mathrm{~m})$ & $M A E_{\theta}(\mathrm{rad})$ \\
\hline RAC & 0.979 & 1.972 & 0.511 & 0.132 \\
\hline POFC & 0.360 & 0.202 & 0.012 & 0.021 \\
\hline
\end{tabular}

The controller parameters of POFC were set as:

$$
\boldsymbol{\Lambda}=\left[\begin{array}{ccc}
16 & & \\
& 16 & \\
& & 4
\end{array}\right], \quad \boldsymbol{K}_{d}=\left[\begin{array}{lll}
1 & & \\
& 1 & \\
& & 1
\end{array}\right] .
$$

The observer parameters of POFC for the $x$ and $y$ channel were set as $\omega_{o 1}=\omega_{o 2}=16 \mathrm{rad} / \mathrm{s}$, and the observer parameter for the rotational channel was set as $\omega_{o 3}=4 \mathrm{rad} / \mathrm{s}$. It should be noted that all of the experiments in this paper were conducted using the same parameters above, without any retuning.

The integral of absolute error (IAE) and maximum absolute error (MAE) were used to evaluate the control performances.

The definition of IAE and MAE are as follows:

$$
\begin{aligned}
& \left\{\begin{array}{l}
I A E_{x y}[m]=\int_{0}^{T}\left(\left|e_{x}\right|+\left|e_{y}\right|\right) d t, \\
I A E_{\theta}[\mathrm{rad}]=\int_{0}^{T}\left|e_{\theta}\right| d t .
\end{array}\right. \\
& \left\{\begin{array}{l}
M A E_{x y}[\mathrm{~m}]=\max \left\{\max \left|e_{x}\right|, \quad \max \left|e_{y}\right|\right\}, \\
M A E_{\theta}[\mathrm{rad}]=\max \left|e_{\theta}\right| .
\end{array}\right.
\end{aligned}
$$

In the first experimental scenario, the robot was commanded to track a circle of $0.8 \mathrm{~m}$ radius within $30 \mathrm{~s}$, i.e., $x_{d}=0.8 \cos \left(\frac{\pi}{15} t\right)$ $\mathrm{m} ; y_{d}=0.8 \sin \left(\frac{\pi}{15} t\right) \mathrm{m}$. In the first $10 \mathrm{~s}$, the robot performed translational motion without rotation, i.e., $\theta_{d}=0 \mathrm{rad}$. After 10 $\mathrm{s}$, the desired robot orientation angle was set as $\theta_{d}=0.35(t-10)$ rad. The robot initial posture was set as $\left[\begin{array}{llll}0.8(\mathrm{~m}) & 0(\mathrm{~m}) & 0(\mathrm{rad})\end{array}\right]^{T}$. Experimental results are shown in Fig. 4. The calculation results of IAE and MAE (steady state) are shown in Table 2.

It is shown in Fig. 4 (b) that the tracking error of the proposed POFC is much smaller than that of model-based RAC in both translational and rotational motion. As is displayed in Table 2, the $I A E_{x y}$ and $I A E_{\theta}$ of RAC are almost 3 times and 10 times as large as those of POFC, respectively. It is also found that the $M A E_{x y}$ and $M A E_{\theta}$ of RAC are almost 5 times and 6 times as large as those of POFC, respectively. This is due to the fact that unmodeled dynamics (i.g., friction forces) and parameter uncertainties exist in the robot control system, which will deteriorate the tracking performances of model-based RAC. However, in the proposed POFC, the unmodeled dynamics and parameter uncertainties can be estimated in the observer (see Fig. 4 (c)) and compensated in the control input (see Fig. 4 (d)).

Moreover, the proposed output feedback design only requires the robot position information, while RAC is a state feedback design requiring both position and velocity information. Emphasis should be paid that velocity signal is obtained by differentiating the position signal in many cases, which may result in deterioration of the control performance due to the introduction of high frequency noise.
Table 3: Robustness Comparisons.

\begin{tabular}{ccccc}
\hline IAE & $I A E_{x y}^{W T}(\mathrm{~m})$ & $I A E_{x y}^{W}(\mathrm{~m})$ & $I A E_{\theta}^{W T}(\mathrm{rad})$ & $I A E_{\theta}^{W}(\mathrm{rad})$ \\
\hline RAC & 0.147 & 0.538 & 0.136 & 0.637 \\
\hline POFC & 0.058 & 0.075 & 0.032 & 0.053 \\
\hline MAE & $M A E_{x y}^{W T}(\mathrm{~m})$ & $M A E_{x y}^{W}(\mathrm{~m})$ & $M A E_{\theta}^{W T}(\mathrm{rad})$ & $M A E_{\theta}^{W}(\mathrm{rad})$ \\
\hline RAC & 0.012 & 0.035 & 0.011 & 0.094 \\
\hline POFC & 0.005 & 0.006 & 0.005 & 0.007 \\
\hline
\end{tabular}

\subsection{Robustness}

In this experimental scenario, a lemniscate trajectory was selected to compare the robustness of the proposed design with RAC. The reference lemniscate trajectory was set as: $x_{d}=0.4$ $\cos \left(\frac{\pi}{40} t\right) \mathrm{m} ; y_{d}=0.4 \sin \left(\frac{\pi}{20} t\right) \mathrm{m}$. In the first $40 \mathrm{~s}$, the robot orientation was fixed, i.e., $\theta_{d}=0 \mathrm{rad}$. After $40 \mathrm{~s}$, the desired orientation angle was $\theta_{d}=\frac{\pi}{40}(t-40) \mathrm{rad}$. The robot initial posture was set as $[0.4(\mathrm{~m}) 0(\mathrm{~m}) 0(\mathrm{rad})]^{T}$. The control parameters of both RAC and POFC were set to be the same as before.

Fig. 5 shows the experimental setup for the robustness test. The experiments were conducted on a rubber floor, to increase friction forces. To introduce payload disturbances, a chair was installed on the robot prototype. In the first $60 \mathrm{~s}$, no payload disturbance was introduced. After $60 \mathrm{~s}$, payload disturbance was introduced by adding a person (weight: $70 \mathrm{Kg}$ ) on the robot prototype (i.e., totally $105 \mathrm{Kg}$ ). In other words, the robot mass has been increased $200 \%$ compared with the original mass (i.e., $35 \mathrm{Kg}$ ). Besides, robot moment of inertia around the mass center $\left(I_{v}\right)$ and friction forces also have a significant change due to the payload disturbance. Therefore, both large parameter uncertainties and large external disturbances were introduced after 60 s. Experimental results are shown in Fig. 6 and Fig. 7. Table 3 also shows the performance index of the two control methods after $60 \mathrm{~s}$. $I A E_{x y}^{W T}, M A E_{x y}^{W T}, I A E_{\theta}^{W T}$ and $M A E_{\theta}^{W T}$ represent the index of performance without payload disturbances. $I A E_{x y}^{W}$, $M A E_{x y}^{W}, I A E_{\theta}^{W}$ and $M A E_{\theta}^{W}$ represent the index of performance with payload disturbances.

It can be seen in Fig. 6 that the control performance of RAC has large deviations after the payload disturbance was introduced (i.e., after $60 \mathrm{~s}$ ). It is also illustrated in Table 3 that $M A E_{x y}$ and $M A E_{\theta}$ of RAC with payload disturbances have respectively increased to $292 \%$ and $855 \%$ compared with RAC without disturbances. However, it can be seen from Fig. 7 and Table 3 that for POFC, the tracking performance remains almost the same after payload disturbance was introduced. As a result, compared with RAC, the proposed POFC achieves much better robustness to large parameter uncertainties and large external disturbances.

\subsection{Discussion}

The experimental results above demonstrate that the proposed POFC enjoys essentially much better robustness to large external disturbances and large parameter uncertainties, yet achieves much better tracking accuracy performances, compared with RAC. It should be emphasized that the superior performance 


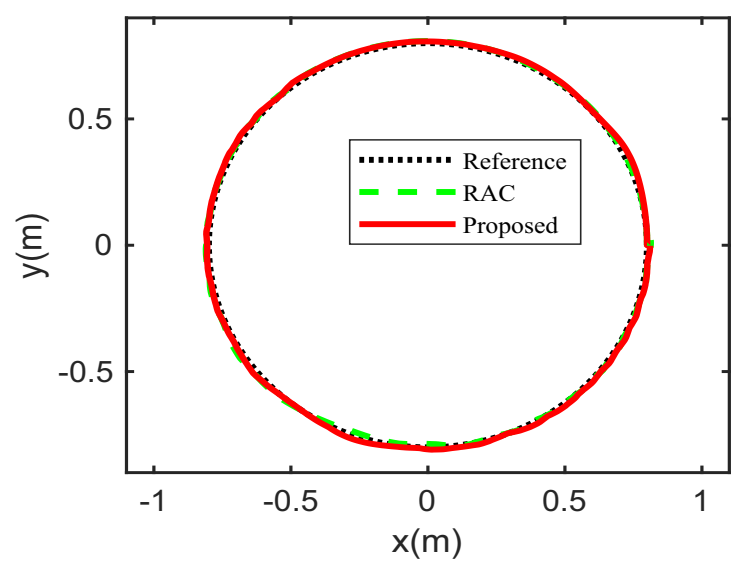

(a)

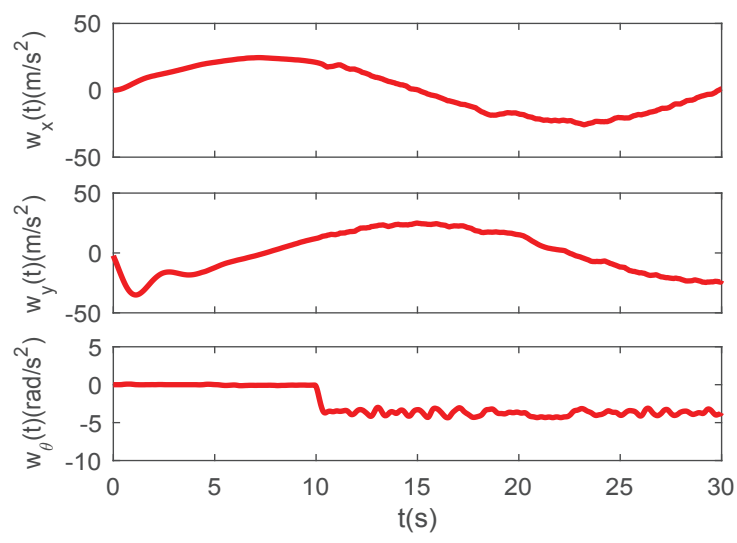

(c)
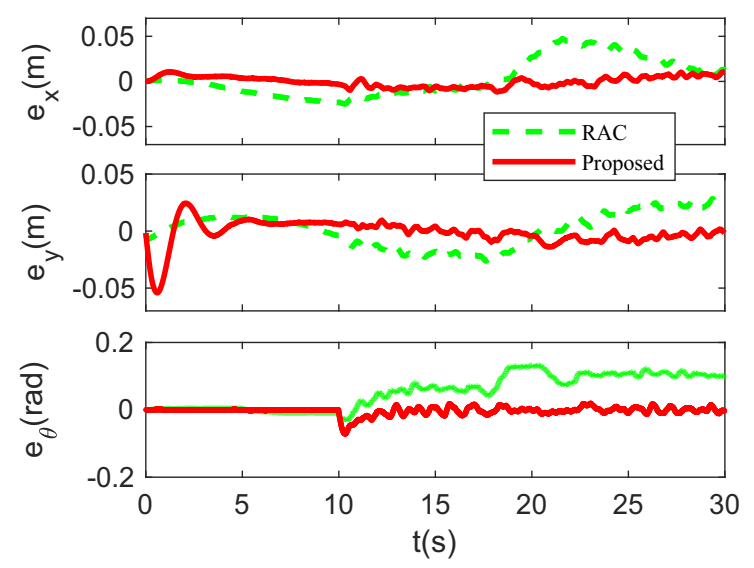

(b)
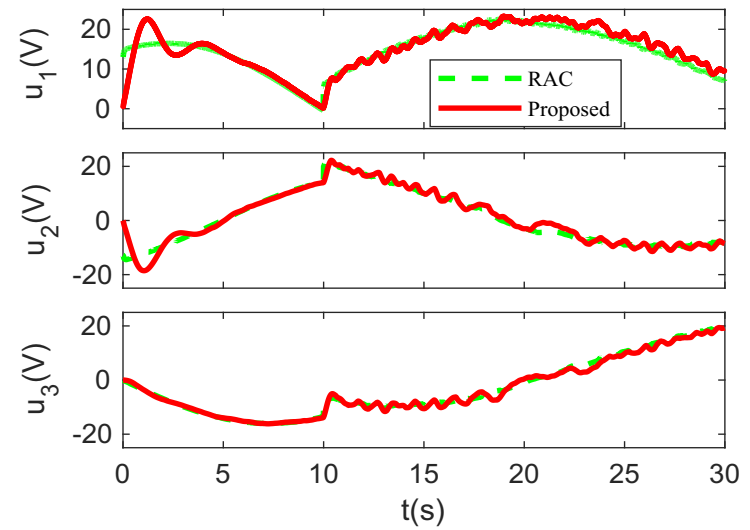

(d)

Figure 4: Experimental results of RAC and POFC (circle trajectory): (a) Reference trajectory and responses in the xy-plane. (b) Tracking errors. (c) Estimated disturbances $\boldsymbol{w}(t)$ by observer. (d) Control input $\boldsymbol{u}(t)$.

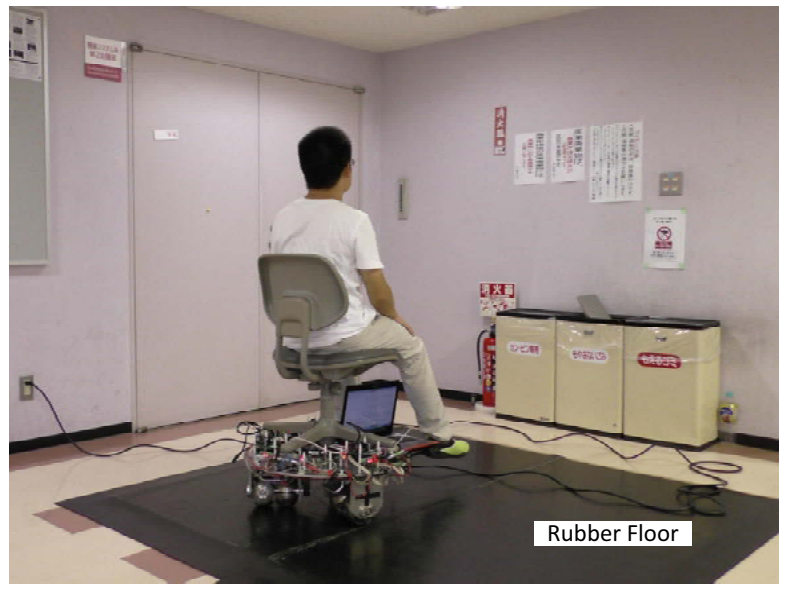

Figure 5: Experimental setup of robust tests. of the proposed POFC is achieved only requiring a geometric parameter, i.e., $L_{0}$, without any other robot model parameter information.

It should be noted that $\boldsymbol{K}_{d}$ in POFC (see (26)) was set considerably small compared with that of RAC (see (25)). Actually, it is shown in simulations and experiments that $\boldsymbol{K}_{d}$ can be set as zero, yet the control performance remains almost unchanged. Therefore, the problems due to large differential feedback, such as introduction of high frequency noise, can be avoided. This is because the proposed controller makes an effective exploitation of the robot natural damping. The robot prototype itself already has large enough damping. However, if the robot natural damping is not large enough, damping should be added in the controller.

For RAC, however, the parameter $\boldsymbol{K}_{d}$ should be selected large to add enough damping, since the natural damping of the robot itself has already been canceled in the controller. If RAC uses the same samll $\boldsymbol{K}_{d}$ as that of POFC, it is found in the experiments that the control system cannot work and have severe oscillations. 


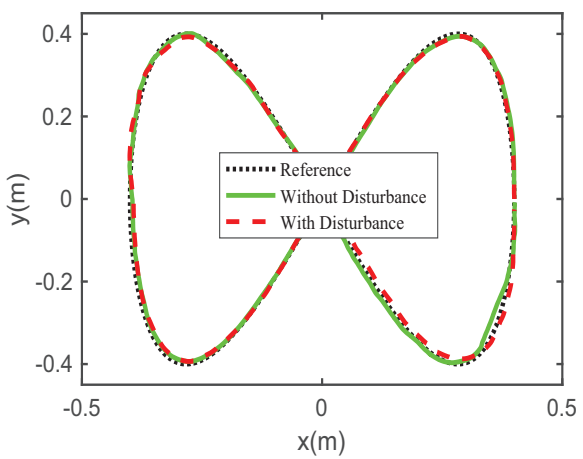

(a)

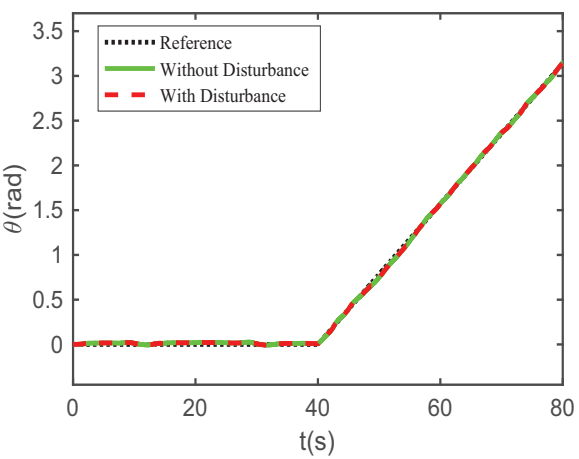

(b)

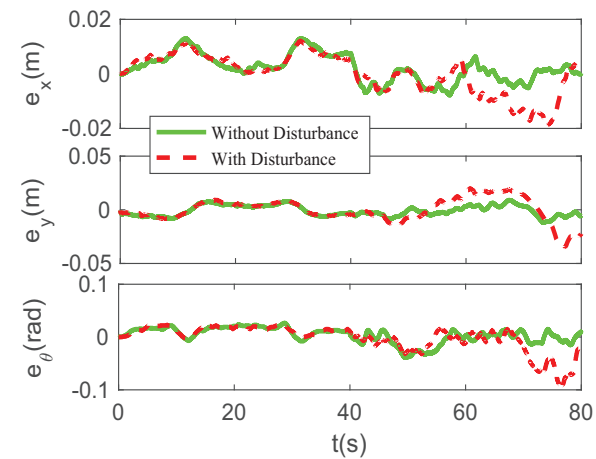

(c)

Figure 6: Experimental results of RAC: (a) Reference trajectory and responses in the xy-plane. (b) Reference trajectory and responses in the rotational direction. (c) Tracking errors.

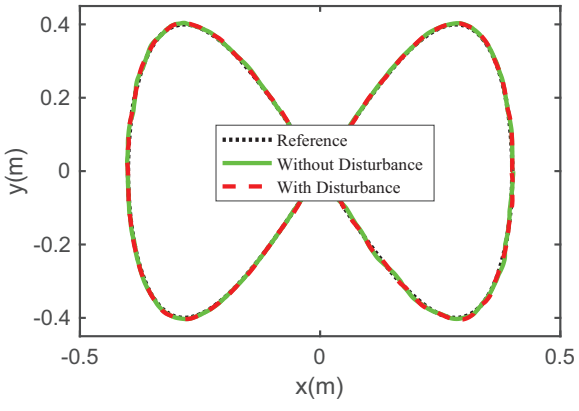

(a)

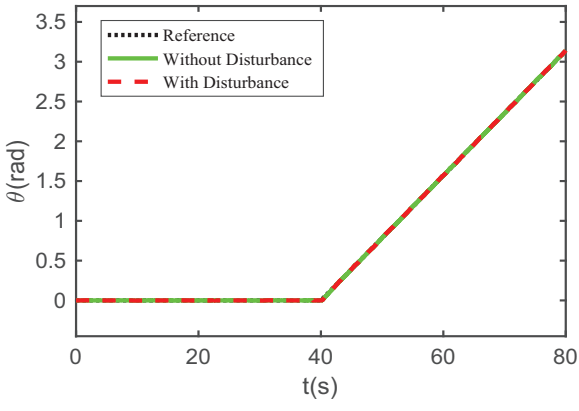

(b)

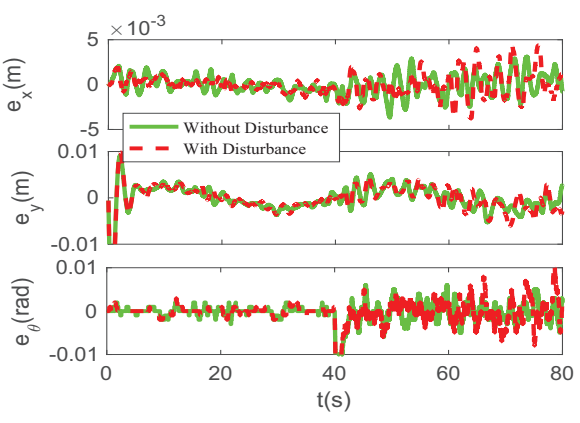

(c)

Figure 7: Experimental results of POFC: (a) Reference trajectory and responses in the xy-plane. (b) Reference trajectory and responses in the rotational direction. (c) Tracking errors.

\section{Conclusions}

In this paper, a passivity-based output feedback tracking control scheme has been proposed for a three-wheeled OMR using only one geometric parameter. Compared with traditional RAC, the proposed control approach does not require any model parameter information except the contact radius. The controller is designed based on a modified GPI observer and passivity property. The design objective is to preserve the passivity property of closed-loop system, which is conceptually different from the traditional control design theoretic methodology. The designed control scheme takes full advantage of the robot natural damping forces, where only considerably small or non differential feedback is needed. Stability analysis has shown that the control system is BIBO stable. Experimental results have demonstrated that the proposed POFC enjoys much better robustness to large external disturbances and large parameter uncertainties, yet achieves much better tracking accuracy performances, compared with RAC.

\section{References}

[1] J.-B. Song and K.-S. Byun, "Design and control of a four-wheeled omnidirectional mobile robot with steerable omnidirectional wheels," J. Robot. Syst., vol. 21, no. 4, pp. 193-208, 2004.

[2] K. Watanabe, Y. Shiraishi, S. Tzafestas, J. Tang, and T. Fukuda, "Feedback control of an omnidirectional autonomous platform for mobile service robots," J. Intell. Robot. Syst., vol. 22, pp. 315-330, 1998.
[3] Y. Liu, J. J. Zhu, R. L. W. II, and J. Wu, "Omni-directional mobile robot controller based on trajectory linearization," Robot. Auton. Syst., vol. 56, no. 5 , pp. $461-479,2007$.

[4] A. Conceicao, A. Moreira, and P. Costa, "Practical approach of modeling and parameters estimation for omnidirectional mobile robots," IEEE/ASME Trans. Mechatronics, vol. 14, no. 3, pp. 377-381, 2009.

[5] J. C. L. Barreto S., A. G. S. Conceicao, C. E. T. Dorea, L. Martinez, and E. R. de Pieri, "Design and implementation of model-predictive control with friction compensation on an omnidirectional mobile robot," IEEE/ASME Trans. Mechatronics, vol. 19, no. 2, pp. 467-476, 2014.

[6] K.-Y. Tu, "A linear optimal tracker designed for omnidirectional vehicle dynamics linearized based on kinematic equations," Robotica, vol. 28, no. 07, pp. 1033-1043, 2010.

[7] J.-T. Huang, T. Hung, and M.-L. Tseng, "Smooth switching robust adaptive control for omnidirectional mobile robots," IEEE Trans. Control Syst. Technol., vol. 23, no. 5, pp. 1986-1993, 2015.

[8] D. Rotondo, V. Puig, F. Nejjari, and J. Romera, "A fault-hiding approach for the switching quasi-LPV fault-tolerant control of a four-wheeled omnidirectional mobile robot," IEEE Trans. Ind. Electron., vol. 62, no. 6, pp. 3932-3944, 2015.

[9] C. Ren, S. Ma, Y. Sun, and C. Ye, "A continuous dynamic modeling approach for an omnidirectional mobile robot," Adv. Robotics, vol. 29, no. 4, pp. 253-271, 2015.

[10] C. Ren and S. Ma, "Generalized proportional integral observer based control of an omnidirectional mobile robot," Mechatronics, vol. 26, pp. 3644, 2015.

[11] R. Comasolivas, J. Quevedo, T. Escobet, A. Escobet, and J. Romera, "Modeling and robust low level control of an omnidirectional mobile robot," J. Dyn. Sys., Meas., Control, vol. 139, no. 4, 2017.

[12] R. Ortega and M. W. Spong, "Adaptive motion control of rigid robots: A tutorial," Automatica, vol. 25, no. 6, pp. 877 - 888, 1989.

[13] J. Zhang and C. C. Cheah, "Passivity and stability of human-robot inter- 
action control for upper-limb rehabilitation robots," IEEE Trans. Robot., vol. 31, no. 2, pp. 233-245, April 2015.

[14] S. F. Atashzar, M. Shahbazi, M. Tavakoli, and R. V. Patel, "A passivitybased approach for stable patient-robot interaction in haptics-enabled rehabilitation systems: Modulated time-domain passivity control," IEEE Trans. Ind. Electron., vol. 25, no. 3, pp. 991-1006, 2017.

[15] L. Sabattini, C. Secchi, B. Capelli, and C. Fantuzzi, "Passivity preserving force scaling for enhanced teleoperation of multirobot systems," IEEE Robot. Autom. Lett., vol. 3, no. 3, pp. 1925-1932, 2018.

[16] M. Shahbazi, S. F. Atashzar, M. Tavakoli, and R. V. Patel, "Position-force domain passivity of the human arm in telerobotic systems," IEEE/ASME Trans. Mechatronics, vol. 23, no. 2, pp. 552-562, April 2018.

[17] H. Sira-Ramírez, C. A. Núñez, and N. Visairo, "Robust sigma-delta generalised proportional integral observer based control of a 'buck' converter with uncertain loads," Int. J. Control, vol. 83, no. 8, pp. 1631-1640, 2010.

[18] A. Rodriguez-Angeles and J. Garcia-Antonio, "Active disturbance rejection control in steering by wire haptic systems," ISA Transactions, vol. 53, no. 4, pp. 939 - 946, 2014.

[19] G. Ramos, J. Cortés-Romero, and H. C.-E. Ram, "Spatial observer-based repetitive controller: an active disturbance rejection approach," Control Eng. Pract., vol. 42, no. 0, pp. 1-11, 2015.

[20] J. Yang, H. Cui, S. Li, and A. Zolotas, "Optimized active disturbance rejection control for dc-dc buck converters with uncertainties using a reduced-order GPI observer," IEEE Trans. Circuits-I, vol. 65, no. 2, pp. 832-841, 2018.

[21] S. Ma, C. Ren, and C. Ye, "An omnidirectional mobile robot: concept and analysis," in Proc. of the 2012 IEEE Int. Conf. Robotics and Biomimetics(ROBIO) , Guangzhou, China, Dec. 2012, pp. 920-925.

[22] C. Ren and S. Ma, "Dynamic modeling and analysis of an omnidirectional mobile robot," in Proc. of the 2013 IEEE/RSJ Int. Conf. on Intelligent Robots and Systems (IROS), Tokyo, Japan, Nov. 2013, pp. 4860-4865.

[23] C. Ren, Y. Sun, and S. Ma, "Passivity-based control of an omnidirectional mobile robot," Robotics $\mathcal{E}$ Biomimetics, vol. 3, no. 1, p. 10, 2016.

[24] J.-J. E. Slotine and W. Li, "On the adaptive control of robot manipulators," Int. J. Robot. Res., vol. 6, no. 3, pp. 49-59, 1987.

[25] R. Ortega, Passivity-based control of Euler-Lagrange systems: mechanical, electrical and electromechanical applications. London, U.K.: Springer, 1998

[26] M. A. Arteaga-Pérez and A. Gutiérrez-Giles, "On the GPI approach with unknown inertia matrix in robot manipulators," Int. J. Control, vol. 87, no. 4, pp. 844-860, 2014.

[27] Y. Chung, C. Park, and F. Harashima, "A position control differential drive wheeled mobile robot," IEEE Trans. Ind. Electron., vol. 48, no. 4, pp. 853-863, 2001.

[28] S. X. Yang, A. Zhu, G. Yuan, and M.-H. Meng, "A bioinspired neurodynamics-based approach to tracking control of mobile robots," IEEE Trans. Ind. Electron., vol. 59, no. 8, pp. 3211-3220, 2012. 